\title{
Do you speak Swiss?
}

Andrée Tabouret-Keller

\section{OpenEdition}

Journals

Édition électronique

URL : http://journals.openedition.org/esp/2821

DOI : 10.4000/esp.2821

ISSN : 2532-0319

\section{Éditeur}

Centre d'Information sur l'Éducation Bilingue et Plurilingue

\section{Édition imprimée}

Date de publication : 1 décembre 2013

Pagination : 95-101

ISSN : 1127-266X

\section{Référence électronique}

Andrée Tabouret-Keller, « Do you speak Swiss? », Éducation et sociétés plurilingues [En ligne], 35 | 2013 mis en ligne le 01 février 2020, consulté le 17 février 2021. URL : http://journals.openedition.org/esp/ 2821 ; DOl : https://doi.org/10.4000/esp.2821 


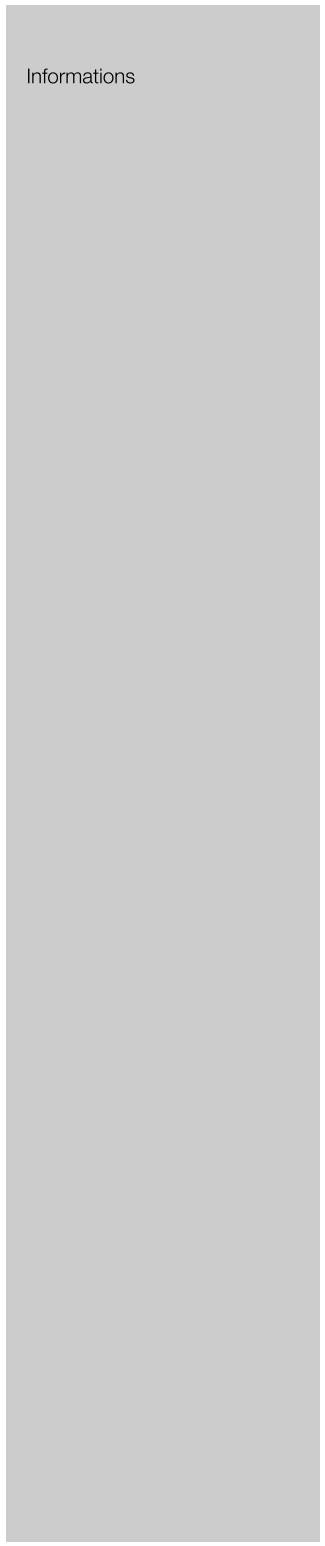

Walter HAAS (éditeur avec le comité de direction), Do you speak Szviss? Zürich, Verlag Neue Zürcher Zeitung, 2010.

\section{Andrée TABOURET-KeLLeR}

Cet ouvrage, entièrement publié en trois langues, le français, l'italien et l'allemand, rend compte des travaux du Programme National de Recherche 56 (PNR 56) dans le cadre du Fond national suisse de la recherche: Diversité des langues et compétences linguistiques en Suisse. La mise en œuvre du projet s'est déroulée entre 2003 et 2009 avec un budget de 8 millions de francs suisses. Un comité de direction international sous la présidence du Professeur Walter Haas a été mis en place dès 2003, la coordination du programme de recherche étant assurée par Ch. Mottaz pour le Programme National. Les PNR sont considérés comme un instrument de la Confédération helvétique pour encourager la recherche en contribuant à élaborer les bases rationnelles de ses politiques.

Le titre de l'ouvrage n'est pas sans un brin d'humour! Le Swiss n'existe pas! La Suisse est un pays plurilingue. La loi sur les langues de 2007 mentionne dans son Article 5 les langues officielles suivantes: l'allemand (plus de $60 \%$ de la population, dont plus de $90 \%$ emploie un dialecte alémanique), le français (autour de $20 \%$ de la population), l'italien (autour de $3 \%$ de la population), langues qui sont utilisées dans leur forme standard par les autorités fédérales (c'est aussi dans cette loi qu'apparaît pour la première fois le terme de «forme standard») mais qui ne sont de loin pas quotidiennement employées sous cette forme; le romanche, la quatrième langue nationale, n'est nommée langue officielle que dans les rapports avec les locuteurs de cette langue et ne connait majoritairement que des emplois dialectaux. Le cas de l'allemand illustre clairement les difficultés liées à la nomination des langues avec un emploi majoritaire de formes dialectales, dont certaines sont d'un emploi dominant dans une portion plus ou moins importante de la population, face à une forme standard («bon allemand», Hochdeutsch, ou Standartdeutsch). Le cas du français est différent: «en Suisse romande il n'existe plus guère de dialectes mais des sociolectes plus ou moins marqués» (p. 52). «La particularité du pays plurilingue qu'est la Suisse réside dans le fait que plusieurs langues y sont officiellement reconnues égales, partiellement ou totalement, alors même que la population qui les parle est de nombre inégal» [...]. Par ailleurs, «au cours des dernières décennies, le quadrilinguisme officiel de la Suisse s'est mué 
en multilinguisme, avec les migrations et la globalisation, et le nombre des minorités y a décuplé» (p. 10). L'hypothèse sur laquelle s'appuie le projet du PNR 56 est «que les acquis politiques et culturels du paysage linguistique doivent être remis sur l'ouvrage en permanence, et que les compétences linguistiques de la population doivent suivre le rythme de l'évolution de la société» (p. 11 ).

L'ouvrage de 240 pages comprend 4 grandes parties: une introduction, un bref aperçu des différents projets, les projets, les perspectives, avec un appendice rendant compte du déroulement du programme de recherche.

Trois grands axes de recherches sont définis (p. 11):

Conditions et cadres juridiques: repenser les bases légales d'une politique cohérente des langues dans un État fédéraliste et multilingue comptant de nouvelles minorités;

Compétences linguistiques: obtenir une vue d'ensemble des compétences linguistiques des hommes et des femmes qui vivent en Suisse, dans leur langue maternelle et dans les langues qu'ils apprennent par la suite, à l'école ou en dehors de l'école;

Langues et identités: examiner le rôle que joue la langue dans l'identité des individus et des communautés.

Une fois le programme de recherche lancé, 90 propositions sont reçues, dont plus de la moitié correspondent à l'axe 2. Après évaluation par un panel de composition internationale, 26 projets de recherche sont retenus, les recherches étant menées à bien de 2006 à 2008, période pendant laquelle plus de 200 conférences, rencontres et communications diverses le font connaître. Les retombées de ce programme se poursuivent jusqu'à aujourd'hui, surtout stimulées par la réflexion critique liée à la mise en valeur des résultats, parfois additionnelle, parfois contradictoire. C'est ainsi que le deuxième chapitre de l'ouvrage «synthèse des résultats», «met en parallèle les résultats des 26 projets en vue d'identifier de nouvelles voies et de nouvelles orientations, mais également des tensions et des contradictions» (p. 46). Les 26 projets font chacun l'objet d'une description donnant son argument, ses recommandations ainsi que le détail des activités de valorisation (publications, formations, interventions) auxquelles il a conduit. Cinq grands questionnements sont identifiés: «comment le plurilinguisme fonctionne», «apprendre le plurilinguisme», «anglais», «variétés standard et non- standard», «minorités». Dans les limites de ce compte rendu, je souligne certains constats qui me paraissent significatifs de la réflexion engagée par la recherche: 


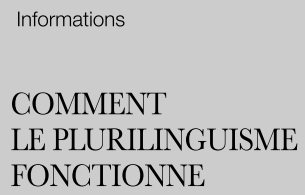

APPRENDRE LE PLURILINGUISME

ANGLAIS
Dans des conditions de fonctionnement au quotidien de groupes dont les membres parlent des langues différentes, l'acquittement d'une tâche commune favorise la compréhension et la collaboration: l'usage qualifié de «flexible» ou «pragmatique» peut être considéré comme point de départ et base de la politique linguistique d'un pays plurilingue, à l'inverse, des règles formelles sont moins efficaces. Cette flexibilité trouve des limites dans la composition des groupes chargés d'élaborer les lois nationales du pays quadrilingue, dans le fait que les tâches à régler exigent suffisamment de personnel dans les quatre langues nationales avec la garantie d'une collaboration améliorée qui devrait faire coïncider les contenus de la communication dans les langues nationales tout en assurant qu'ils fonctionnent dans les différentes cultures (mon commentaire: il est indispensable de l'affirmer mais c'est un idéal dont la réalisation reste problématique). Un autre aspect clairement souligné est celui de la nécessité d'une formation linguistique continue, tant bilingue que plurilingue, en particulier dans le milieu enseignant. Dans les milieux économiques, le plurilinguisme des personnels devrait d'avantage être identifié et mis à profit.

L'école assume l'essentiel du fardeau de la transmission des langues, reste que la définition et le nombre des langues étrangères qui doivent être enseignées par l'école fait débat. Le choix de deux langues étrangères à l'école primaire, quoique accepté, fait l'objet de craintes - la surcharge des élèves - que les enquêtes réalisées permettent d'écarter. La plupart des cantons de Suisse allemande choisissent l'anglais comme langue initiale (contre le français dans les autres cantons alémaniques et le Tessin, l'allemand dans les cantons romands). Le choix de l'anglais précoce fait l'objet de discussions car il entre en concurrence avec les plus petites langues nationales en tant que langues scolaires et mérite d'autres travaux de recherche sur le long terme. La question de l'âge reste elle aussi en débat (acquisition bilingue ou bien apprentissage précoce et successif), de même l'attitude face à l'apprentissage (place donnée aux règles formelles ou bien aux exercices de «pure» communication (p. 50), enfin la place de l'enseignant en tant que personne.

Sa fonction comme lingua franca donne lieu à débat: considéré comme «langue de la jeunesse», c'est un stéréotype dont on risque de surestimer les effets positifs: «les élèves tendraient à séparer distinctement leur vie scolaire et leur vie privée» (p. 50). 

Informations
VARIÉTÉS
STANDARD
ET NON STANDARD

«Pour nombre de migrants la lingua franca la plus logique reste une langue officielle suisse, comme le français pour les ressortissants africains, l'italien pour les albanophones» (p. 51).

La problématique de la diglossie entre ces variétés n'est pas uniforme: «La problématique de la diglossie suisse allemande s'exprime déjà dans le simple fait qu'environ la moitié des personnes interrogées lors d'un sondage ont indiqué comme 'première langue étrangère' l'allemand standard, obligeant à apporter d'importantes corrections à la base de données utilisée pour l'exploitation statistique» (p. 5l). Inversement, le français standard est jugé de manière positive par un tiers des personnes interrogées. «La principale conclusion à retenir est que les habitants des deux régions linguistiques, y compris les jeunes et les individus des couches moyennes inférieures, maîtrisent les variétés et les registres dans la vie quotidienne en fonction de la situation» (p. $52)$.

Un certain nombre de problèmes sont soulignés: la représentation des petites communautés n'est pas équilibrée dans les domaines de la législation plurilingue, ni de la représentation proportionnelle des langues dans l'administration fédérale, ni dans celui de l'enseignement dans les petites langues nationales. On exige des membres des communautés autochtones d'avantage d'efforts linguistiques pour assurer leur place dans les groupes linguistiques mixtes. Une tâche pédagogique importante en résulte: la manière contraignante à l'extrême que peut revêtir l'apprentissage d'une langue étrangère devrait pouvoir être vécue de manière positive (p. 53). La situation des minorités des nouveaux groupes linguistiques dont la langue n'est pas reconnue par la Constitution est préoccupante: alors que les minorités autochtones exigent d'être protégées d'une dissolution dans des groupes plus larges, les minorités allochtones sont traitées de manière opposée dans la mesure où le mot clé qui décide de leur avenir est celui d'intégration: les premiers doivent pouvoir rester distincts de la majorité qui les entoure, les seconds doivent s'y fondre. Le terme d'intégration est à lui seul source de multiples interrogations sur les critères qui pourraient le définir, ces derniers étant loin d'être circonscrits (p. 53). On pense à la place à réserver à la langue d'origine, à l'exploitation des capacités linguistiques des élèves dans leur vie extrascolaire, par exemple leurs compétences en lecture, tant des adultes que des élèves, et leur compétence à une communication plus large (p. 54). 
Informations

LANGUES ET MÉDIAS
Plusieurs problèmes sont précisés: comment les journalistes peuvent-ils «remplir le mandat de droit public des médias officiels qui consiste à encourager l'intégration sociale par une compréhensibilité générale, tout en touchant réellement leur public»? Comment encore tenir compte à la fois du fait que les régions possèdent réellement des cultures politiques différentes et des mentalités politiques propres, héritées de leur histoire, et que par conséquent l'influence des discours médiatiques n'est pas le même? Il semble que les médias ne thématisent et n'interprètent pas un problème de manière semblable selon la région linguistique à laquelle ils appartiennent: de manière autosuffisante, ils ne seraient tenus que par leur «territoire linguistique» et seraient anxieux de ne pas imposer d'autres langues nationales à leur public (p. 55).

Dans l'ouvrage, cette revue des grandes questions posées au PNR56 est suivie par une concise mais précise présentation des 26 projets qui ont fait l'objet d'un travail de recherche. Il n'est guère possible d'en faire le compte rendu ici. Par contre, il est possible de mentionner les principales perspectives qui s'en dégagent. Un rappel d'abord: en prescrivant une thématique, un PNR fait de la recherche appliquée dans la mesure où il propose des thèmes "qui réclament d'urgence un examen scientifique» ( $p$. 151). En ce qui concerne l'objectif idéal dans le cas du PNR56, il s'agit de «favoriser la compréhension et la cohésion entre les groupes linguistiques et d'encourager les identités linguistiques des habitants ainsi que leurs compétences dans une ou plusieurs langues» (p. 151), objectif qui s'inscrit dans les principes fondamentaux de la politique des langues du Conseil de l'Europe affirmés dans Un cadre européen commun de référence pour les langues (GRCRL, Conseil de l'Europe, 2001). Ge dernier souligne que la diversité linguistique et culturelle en Europe «au lieu d'être un obstacle à la communication, devienne une source d'enrichissement et de compréhension réciproque» (GECRL, p. 10). Mais comment traduire ces principes idéaux dans des réalités sociales, s'interrogent avec pertinence les responsables du PNR 56.

La plupart des soucis exprimés dans les perspectives qui clôturent l'ouvrage découlent de la manière dont les projets ont été conçus et dont, dans leur ensemble, ils ont favorisé certains aspects du plurilinguisme suisse ou, au contraire, les ont ignorés. Pour le lecteur, ces remarques sont particulièrement stimulantes: l'examen critique des projets, fait dans l'après-coup, et la liberté des réflexions qui l'accompagnent prennent leur relief dans le contexte de la Suisse dont l'exiguité, par comparaison aux grands 
États voisins, permet sans doute cet exercice; malgré ces différences, ils peuvent faire office de modèle.

Si dans leur ensemble, les projets s'intéressent aux situations à plurilinguisme dominant, quelque-soit par ailleurs ses particularités, l'encouragement et la promotion d'un plurilinguisme fonctionnel, en particulier par le soutien empirique aux mesures scolaires qui le favorisent, semble insuffisant; il convient d'identifier les points névralgiques des situations de contact en surmontant le manque de collaboration et de recherche comparée au-delà des frontières linguistiques; d'encourager la pratique des langues après l'école; de favoriser le dépassement de frontières linguistiques surtout quand elles coincident avec des tendances au nationalisme, tâche qui n'est pas suffisamment exigée des grandes communautés mais l'est particulièrement de la part des plus petites communautés linguistiques (p. 152). Un autre type de frontière linguistique est également visée, c'est celui qui sépare les disciplines et qui touche à la promotion de l'interdisciplinarité dont on déplore le peu d'impact dans les projets. Il serait fondamental de mener une discussion interdisciplinaire ouverte sur la problématique du plurilinguisme, des variétés et des minorités, à condition d'aller au-delà des formules incantatoires et de considérer les champs de tension réels (p. 153), par exemple le fait que les problèmes juridiques attachés aux langues indigènes sont insuffisamment abordés. Par ailleurs, «les efforts visant à surmonter les frontières linguistiques de toute nature se heurtent vite au désir d'identité linguistique des individus, voire aux revendications, reconnues à l'échelle européenne, d'encouragement de toutes les 'variétés' et de reconnaissance de leur dignité» (p. 153).

Un autre objet de souci concerne la limitation de l'encouragement des langues à l'école et aux institutions de formation spécialisées: «Pour beaucoup, le plurilinguisme de l'État signifie le droit de l'individu de ne pas avoir à prendre conscience de l'existence des autres langues. Il s'ensuit une fétichisation de la traduction et l'efficience de l'enseignement des langues à l'école d'une part, et d'autre part le fait que l'encouragement des compétences linguistiques ne saurait se limiter à l'école. Les possibilités de promotion dans la vie quotidienne sont quasiment inépuisables [...]. Or aucun des projets n'a sondé les chances, exploitées ou manquées, de l'éveil aux langues dans la vie quotidienne (p. 154). Que peut englober la notion de vie quotidienne: la clarification des interactions sociales - j'ajoute à la fois complexes et hétérogènes - représente une énorme tâche empirique et un défi théorique. La difficulté du travail de recherche proprement dit est soulignée: com- 
ment le citoyen moyen peut-il s'en faire une idée et ne pas formuler face aux scientifiques des demandes élevées et souvent impatientes, en particulier dans le domaine des sciences humaines? Comment passer du langage des chercheurs et même dans un temps décalé à celui du grand public et des autorités? Et comment passer encore des résultats de la recherche aux applications politiques? In fine, l'on partage le regret de la limitation temporelle d'un PNR: dans le domaine des situations linguistiques, des études longitudinales s'imposent.

L'exposé que nous a fait lors du dernier Samedi matin du CIEBP à Paris le 16 mars 2013, Jean-François De Pietro («Quelques approches originales pour une didactique du plurilinguisme») illustre le programme ÉOLE, Éducation et ouverture aux langues de l'école: il peut être considéré comme une application importante dans la direction des recommandations du PNR 56. Nous en publierons le texte dans un prochain numéro de notre revue.

\section{Penelope GARDNER GHLOROS, Code switching. Cambridge: Cambridge University Press, 2009. Michael CLYNe (1)}

Readers of this journal are well aware of the explosion which the area of bi-/multilingualism/language contact has undergone in the past two decades. There are numerous new journals, conferences, handbooks and textbooks, and masses of monographs in the area. A central aspect of this study - one not clearly defined is code switching. So why do we need a new book entitled Codeswitching? It is a critical synthesis of the field, very accessible to specialists, linguists from other fields, and students being initiated into the field; a book that points the way to new approaches for decades to come - just what is needed at this time.

Penelope Gardner Chloros (hereafter GG) has contributed empirical sociolinguistic and descriptive linguistic studies to the literature on language contact for well over 20 years and is best known for her research on Alsatian-French bilingualism in Strasbourg and on Greek-English bilingualism in London. She is not one of the grand theorists of the field but has made available her innovative ideas, energy and experience to many collaborative ventures including the European Science Foundation Network on Code Switching (CS) and the LIDES bilingual data base project. GC's volume, perhaps more than any other, is characterized by an open-mindedness - not being wedded to any school or paradigm 